\title{
c-Maf inducing protein inhibits cofilin-1 activity and alters podocyte cytoskeleton organization
}

\author{
LIXIA YU ${ }^{1,2}$, JIANMING YE ${ }^{2}$, QIFENG LIU ${ }^{2}$, JIANHUA FENG ${ }^{2}$, XIAOXIA GU ${ }^{2}$, QIANG SUN ${ }^{2}$ and GUOYUAN LU ${ }^{1}$ \\ ${ }^{1}$ Department of Nephrology, The First Affiliated Hospital of Soochow University, Suzhou, Jiangsu 215006; \\ ${ }^{2}$ Department of Nephrology, The First People's Hospital of Kunshan, Kunshan, Jiangsu 215300, P.R. China
}

Received September 1, 2016; Accepted June 6, 2017

DOI: $10.3892 / \mathrm{mmr} .2017 .7156$

\begin{abstract}
The glomerular visceral epithelial cells, also termed podocytes, are key in maintaining the normal renal filtration barrier. Although it has been demonstrated that stimulation of c-Maf inducing protein (CMIP) expression is involved in podocyte damage, the molecular events during this process remain unclear. In the current study, CMIP-induced proximal signaling was investigated by focusing on its effect on cofilin-1 activity in puromycin aminonucleoside (PA)-damaged podocytes. An obvious elevation of CMIP expression and phosphorylated (p) cofilin-1 levels was detected in cultured podocytes treated with PA and in glomeruli isolated from PA-induced nephropathy rats. Stable knockdown of CMIP prevented upregulation of p-cofilin-1 and reorganization of actin cytoskeleton in PA-treated podocytes. The activity of the Src family kinase Fyn was reduced, whereas small GTPase Ras homolog gene family, member A (RhoA) activity was increased in PA-treated podocytes. Stimulation of CMIP expression inhibited Fyn activation and decreased the expression level of p-p190RhoGAP, a negative regulator of RhoA activity. The level of p-LIM domain kinase 1 (LIMK1), a downstream effector of RhoA, increased significantly in PA-treated podocytes. Notably, the applications of RhoA inhibitor or knockdown of LIMK prevented increase of the p-cofilin-1 level in PA-treated podocytes. Thus, the current data provided evidence that the CMIP/Fyn/RhoA/cofilin-1 signaling pathway may be associated with actin disorganization and podocyte foot process spreading following podocyte injury.
\end{abstract}

Correspondence to: Dr Jianming Ye, Department of Nephrology, The First People's Hospital of Kunshan, 91 Qianjin West Road, Kunshan, Jiangsu 215300, P.R. China

E-mail:ks_yjm@163.com

Dr Guoyuan Lu, Department of Nephrology, The First Affiliated Hospital of Soochow University, 188 Shizi Street, Suzhou, Jiangsu 215006, P.R. China

E-mail: luguoyuan@medmail.com.cn

Key words: podocyte, c-Maf inducing protein, Ras homolog gene family, member, cofilin-1, actin remodeling, puromycin aminonucleoside

\section{Introduction}

Proteinuria, one of the most important manifestations in renal glomerular diseases, results from the dysfunctional glomerular filter, particularly the slit diaphragm, which is a specialized structure interdigitating podocyte foot processes (1). The podocyte is a terminally differentiated epithelial cell soaked in the urinary space and attached to the glomerular basement membrane by large cell protrusions, termed foot processes (2). As the ultimate layer of glomerular filtration barrier, podocytes and the slit diaphragm are crucial in maintaining the normal function of renal filtration (2). Following podocyte injury, cytoskeletal disorganization-mediated effacement of foot processes and damage to the slit diaphragm may be a major causative pathogenesis in podocytes, accounting for the occurrence and development of proteinuria $(3,4)$.

c-Maf-inducing protein (CMIP), was initially identified in $\mathrm{T}$ cells of patients with minimal change disease, exhibiting increased expression levels in these patients (5). B7-1, an immune-associated costimulatory protein necessary for T-cell activation and survival, was expressed in podocytes. It has been reported that increased B7-1 is associated with the development of proteinuria in human and murine lupus nephritis, in nephrin knockout mice and in lipopolysaccharide-injected mice $(6,7)$. Similarly, a very low abundance of CMIP was present in podocytes (5). The specific transgenic mice, with CMIP present in podocytes, demonstrated massive proteinuria and developed foot process effacement (5). Expression levels of CMIP were observed to be increased in passive-type Heymann nephritis and adriamycin (ADR)-induced nephropathy $(8,9)$. In addition, the abundance of CMIP was enhanced in interleukin (IL)-17-induced podocyte damage (9). Stimulation of CMIP expression levels disrupted the interaction between the Src kinase Fyn and the cytoskeletal regulator, neural Wiskott-Aldrich syndrome, and between the adaptor protein, Nck and the key slit diaphragm protein, nephrin (5). This indicates that CMIP is critical in regulating the remodeling of actin cytoskeleton responsible for foot process effacement, while the underlying molecular mechanisms require further investigation.

Cofilin, a family of actin-binding proteins, regulates the assembly and disassembly of actin filaments $(10,11)$. Cofilin exists in three highly conserved isoforms termed cofolin-1, cofilin-2 and destrin or actin depolymerizing factor (11). 
Cofilin-1 is predominantly expressed in non-muscle cells (11). Podocyte cofilin-1 knockout mice developed persistent proteinuria by the age of 3 months, and exhibited foot process until 18 months of age, with more exaggerated proteinuria (12). Thus, cofilin-1 is required for maintenance of normal podocyte architecture and for actin structural changes that occur during induction and recovery from podocyte injury $(13,14)$.

As CMIP and cofilin-1 are associated with the regulation of podocyte actin cytoskeleton, the functional association between CMIP and cofolin-1 were investigated in the current study, and stimulation of CMIP expression resulted in actin disorganization via downregulation of cofilin-1 activity in puromycin aminonucleoside (PA)-induced podocyte damage.

\section{Materials and methods}

Antibodies. The following primary antibodies were used in the current study: Mouse anti-cofilin-1 (ab54532), rabbit anti-p-(Ser3)-cofilin-1 (ab131274), rabbit anti-p-(Tyr1105)-p190RhoGAP (growth regulating factor 1; GRF1; ab182636), mouse anti-LIM kinase 1 (LIMK1; ab119084), mouse anti-Fyn (ab1881), and rabbit anti-p-(Thr508)-LIMK1 antibody (ab194798; all from Abcam, Cambridge, MA, USA); rabbit anti-CMIP (12851-1-AP; ProteinTech Group, Inc., Chicago, IL, USA); rabbit anti-GRF1 polyclonal antibody (PA5-38253; Pierce; Thermo Fisher Scientific, Inc., Waltham, MA, USA); rabbit anti-Src ${ }^{\text {Tyr418 }}$ antibody (OPA1-03091; Invitrogen; Thermo Fisher Scientific, Inc.); rabbit anti-Src ${ }^{\text {Tyr527 }}$ antibody (2105; Cell Signaling Technology, Inc., Danvers, MA, USA); mouse anti-GAPDH (G8795; Sigma-Aldrich; Merck KGaA, Darmstadt, Germany) and mouse anti- $\beta$-actin (A5441; Sigma-Aldrich; Merck KGaA) antibodies.

PA nephrosis establishment. A total of 12 male Wistar rats, weighing 100-120 g were i.p. injected with Sigma-Aldrich PA (10 mg/100 g body weight; Merck KGaA) or an equal volume of saline control. A 24-h urine sample was collected from each rat, and proteinuria was measured on an automatic biochemical analyzer (7170A, Hitachi, Ltd., Tokyo, Japan) (15). At day 0, 7 and 15 following the injections, all rats were sacrificed under ether anesthesia according to the Guide for the Care and Use of Laboratory Animals at the Kunshan First People's Hospital (Kunshan, China). Rat glomeruli were isolated using a typical sequential sieve $(150,100$ and $75 \mu \mathrm{m})$ and stored at $-80^{\circ} \mathrm{C}$ for further protein extraction.

Mouse podocyte culture and treatment. The mouse podocyte cell line was provided by Professor Peter Mundel (Massachusetts General Hospital and Harvard Medical School, Boston, MA, USA), and cultured at $33^{\circ} \mathrm{C}$ for proliferation in Gibco RPMI-1640 media (Thermo Fisher Scientific, Inc.) supplemented with Gibco $10 \%$ fetal calf serum (Thermo Fisher Scientific, Inc.), $100 \mathrm{U} / \mathrm{ml}$ penicillin/streptomycin and $10 \mathrm{U} / \mathrm{ml}$ recombinant mouse interferon (IFN) $-\gamma$ (Sigma-Aldrich; Merck $\mathrm{KGaA}$ ). Cells were subsequently moved to $37^{\circ} \mathrm{C}$ and cultured for 14 days to induce differentiation by removal of IFN- $\gamma$. Podocytes were treated with $75 \mu \mathrm{g} / \mathrm{ml} \mathrm{PA}$ for 12,24 and $48 \mathrm{~h}$. The small GTPase Rho inhibitor I (CT04-A; Cytoskeleton, Inc., Denver, CO, USA) was used at concentrations of 0.5 and
$1.0 \mathrm{mg} / \mathrm{ml}$ to explore the role of RhoA in PA-induced podocyte damage. Podocytes overexpressing pcDNA-CMIP (a kind gift from Professor Dil Sahali, Inserm U955, Paris, France) were used in the present study.

Short interfering RNA (siRNA). pSilencer 2.1-U6 (Ambion; Thermo Fisher Scientific, Inc.) was used to express siRNAs that specifically target mouse CMIP and LIMK. The validated siRNA target sequences were obtained from Sigma-Aldrich and were as follows: siCMIP, 5'-gaggatccttaaacataat-3'; si-LIMK, 5'-gctaaacttcatcacagagtac-3' (Merck KGaA). Mouse podocytes were transfected with the above-mentioned siRNA plasmids using Lipofectamine 2000 (11668027; Invitrogen; Thermo Fisher Scientific, Inc.). After 48 h, to establish stable knockdown cells, cells were selected with neomycin $(500 \mu \mathrm{g} / \mathrm{ml})$ until resistant colonies of cells appeared.

Reverse transcription-quantitative polymerase chain reaction $(R T-q P C R)$. Total RNA was extracted from the rat kidney cortex and cultured podocytes using TRIzol reagent (Thermo Fisher Scientific, Inc.). RNA $(1.5 \mu \mathrm{g})$ was used for cDNA synthesis (Thermo Fisher Scientific, Inc.) and $2 \mu \mathrm{l}$ cDNA was used to perform qPCR with 1X SYBR Green Mix (Bio-Rad Laboratories, Inc., Hercules, CA, USA) and $250 \mathrm{nM}$ of primers on a 7700 real-time PCR Detection System (Applied Biosystems; Thermo Fisher Scientific, Inc.). The following primer sequences were used: Sense, $5^{\prime}$-gctgcttgggagcgacgtgt-3' and antisense, 5'-cgttaggtggtgcggctccc-3' for CMIP; sense, 5'-caaggatgccatcaagaa-3' and antisense, 5'-gtccttgacctcctcgta-3' for cofilin-1; and sense, 5'-tgtgtccgtcgtggatctga-3' and antisense, 5'-cctgcttcaccaccttcttga-3' for GAPDH. The PCR thermocycling program was started with initial denaturation at $95^{\circ} \mathrm{C}$ for $1 \mathrm{~min}$, followed by 40 cycles of denaturation at $95^{\circ} \mathrm{C}$ for $15 \mathrm{sec}$, annealing and extension at $60^{\circ} \mathrm{C}$ for $30 \mathrm{sec}$. The normalized mRNA gene level was calculated using $2^{-\Delta \Delta \mathrm{Cq}}$ and standardized to control (16).

Actin cytoskeleton staining and quantification. Podocytes were cultured on coverslips in 12-well plates. Cells were fixed with $4 \%$ paraformaldehyde, prepared in phosphate-buffered saline (PBS) and permeabilized with $0.25 \%$ Triton X-100/PBS (Sigma-Aldrich; Merck KGaA). Non-specific staining was reduced by incubation in $3 \%$ bovine serum albumin (BSA)/PBS at room temperature for $30 \mathrm{~min}$. Actin cytoskeleton was revealed with Alexa 488-conjugated phalloidin. After three washes with PBS, the coverslips were mounted on glass slides with immunofluorescence anti-fade reagent (Invitrogen; Thermo Fisher Scientific, Inc.). Images were obtained using immunofluorescence microscopy and processed with Photoshop software (Adobe Systems, Inc., San Jose, CA, USA). Actin filaments were analyzed using image thresholding techniques using the Image $1.49 \mathrm{p}$ plugin, Analyze Particles (National Institutes of Health, Bethesda, MD, USA). The lengths of actin filaments, defined as Feret's diameter (the longest distance between any two points along the selection boundary) were measured, and the average length (mean \pm standard deviation) of actin filaments was compared.

Immunoblotting and immunoprecipitation assay. Total glomerular and cellular protein was extracted using RIPA 
buffer (1\% Nonidet P-40, 0.1\% SDS, $0.5 \%$ deoxycholate, $1 \mathrm{mM}$ EGTA (pH 8.0) and 10\% glycerol) supplemented with protease and phosphatase inhibitors (Roche Diagnostics, Indianapolis, IN, USA). Protein concentration was quantified using a bicinchoninic acid assay kit (Bio-Rad Laboratories, Inc.). In total, $75 \mu \mathrm{g}$ protein was used for 7.5 and $12.5 \%$ SDS polyacrylamide gel electrophoresis (90 V for 1-1.5 h), and the protein was transferred to nitrocellulose membranes (GE Healthcare Bio-Sciences, Pittsburgh, PA, USA). The membrane was blocked for $1 \mathrm{~h}$ at room temperature in $5 \%$ BSA/Tris-buffered saline containing $0.05 \%$ Tween-20 (TTBS). The following primary antibodies were diluted in 5\% BSA/TTBS and incubated at $4^{\circ} \mathrm{C}$ overnight: Rabbit anti-CMIP (1:500), rabbit anti-p-cofilin-1 (1:1,000), mouse anti-cofilin-1 (1:1,000), mouse anti-LIMK1 (1:400), rabbit anti-p-LIMK1 (1:600), rabbit anti-p-GRF1 (1:800), rabbit anti-GRF1 (1:500), mouse anti-Fyn (1:1,000), rabbit anti-Src ${ }^{\text {Tyr418 }}(1: 750)$, rabbit anti-Src ${ }^{\text {Tyr527 }}$ (1: 1,000 mouse anti-GAPDH $(1: 5,000)$, and mouse anti- $\beta$-actin $(1: 10,000)$. After five washes with TTBS, the membrane was incubated with horseradish peroxidase-conjugated goat anti-mouse or rabbit IgG (1:5,000 in 5\% BSA/TTBS; Invitrogen; Thermo Fisher Scientific, Inc.) for $1 \mathrm{~h}$ at room temperature. The blots were developed with an enhanced ECL chemiluminescence detection kit (Pierce; Thermo Fisher Scientific, Inc.), and the specific band was scanned and quantified using ImageJ version 1.47 (National Institutes of Health, Bethesda, MD, USA).

To analyze the specific activity of the Src kinase, Fyn, an immunoprecipitation assay was performed. Briefly, a total of $250 \mu \mathrm{g}$ protein was incubated with $2 \mu \mathrm{g}$ mouse anti-Fyn antibody overnight at $4^{\circ} \mathrm{C}$ and $2 \mu \mathrm{g}$ of normal mouse IgG (sc-2025; Santa Cruz, Shanghai, China) served as a control. Protein $\mathrm{G}$ agarose beads $(50 \mu \mathrm{l})$ were added and incubated for $4 \mathrm{~h}$. Following three washes by centrifugation $\left(3,000 \mathrm{xg}\right.$, at $4^{\circ} \mathrm{C}$ for $1 \mathrm{~min}$ ), $20 \mu \mathrm{l}$ of $2 \mathrm{X}$ loading buffer was added to the beads and boiled for $5 \mathrm{~min}$. Finally, an immunoblot assay as described above was performed using the anti-pSrc418 antibody.

RhoA activity detection. The Rho switch operates by alternating between an active guanosine-5'-triphosphate (GTP)-bound state and an inactive guanosine diphosphate (GDP)-bound state. The Raf-like Ras-binding domain (RBD) motif of the Rho effector protein, Rhotekin, has been demonstrated to bind specifically to the GTP-bound form of RhoA. Therefore, glutathione S-transferase-tagged Rhotekin-RBD protein on colored agarose beads (Cytoskeleton, Inc.) was used to quantify RhoA activation in cells Briefly, $200 \mu \mathrm{g}$ samples were combined with $20 \mu \mathrm{l}$ reconstitute Rhotekin-RBD beads, and gently rotated at $4^{\circ} \mathrm{C}$ for $1 \mathrm{~h}$. Beads were then washed five times in Wash Buffer (25 mM Tris ( $\mathrm{pH} 7.5), 30 \mathrm{mM}$ $\mathrm{MgCl}_{2}$ and $40 \mathrm{mM} \mathrm{NaCl}$ ) by centrifugation $\left(3,000 \mathrm{x}\right.$, at $4^{\circ} \mathrm{C}$ for $1 \mathrm{~min})$. Finally, bead pellets were resuspended in $30 \mu \mathrm{l}$ of 2X SDS Lamelli buffer (1610737; Bio-Rad Laboratories, Inc.) supplemented with $20 \mathrm{mM}$ DTT and boiled for $5 \mathrm{~min}$. GTP $\gamma \mathrm{S}$ - and GDP-treated samples served as positive and negative controls, respectively.

Statistical analysis. Data are presented as the mean \pm standard deviation. Statistical evaluation was performed using one- or two-way analysis of variation and $\mathrm{P}<0.05$ was considered to indicate a statistically significant difference.

\section{Results}

Stimulation of CMIP expression is associated with cofilin-1 activity downregulation and actin reorganization in PA-induced podocyte damage. The alteration of CMIP and cofilin-1 expression levels was assessed in PA-induced podocyte damage in vitro and in vivo. At the indicated time points, no marked changes in total cofilin-1 abundance were observed at the mRNA and protein expression levels following PA treatment in cultured mouse podocytes (Fig. 1A and B; P>0.05). However, the immunoblot assay detected a time-dependent significant increase of $\mathrm{p}$-cofilin- ${ }^{\mathrm{Ser} 3}$ in PA-treated podocytes compared with untreated cells (Fig. 1B; $\mathrm{P}<0.01$ ). The mRNA and protein abundance of CMIP significantly increased $(\mathrm{P}<0.01)$ in a time-dependent manner in PA-treated podocytes (Fig. 1A and B). Nephrosis was induced in rats by i.p. injection of PA (10 mg/100 g body weight), and 24-h proteinuria was analyzed. Compared with day 0 , proteinuria was significantly detected at day 3 , peaked at day 7 , and then decreased at day 14 following the PA injections (Fig. $1 \mathrm{C} ; \mathrm{P}<0.01$ ). Immunoblotting of the isolated glomeruli indicated that CMIP mRNA and protein expression levels were significantly increased at days 3 and 7 in PA-injected rats (Fig. 1D and E). Total cofilin-1 did not change, whereas p-cofilin- $1^{\mathrm{Ser} 3}$ increased significantly $(\mathrm{P}<0.01)$ at days 3 and 7 following the PA injections (Fig. 1F). Notably, the increased p-cofilin-1 expression level indicates a reduction of cofilin-1 activity (11). Thus, increased CMIP and reduced cofilin-1 activity may be associated with PA-induced podocytes.

To assess the potential association between CMIP and cofilin-1, knockdown of CMIP was performed using the specific siRNA against the mouse CMIP gene (siCMIP) and the podocyte clone, which stably expresses siCMIP, was obtained by selection with neomycin. As compared with control siRNA (siCTL), the CMIP protein expression level was significantly decreased in non-PA-treated podocytes expressing siCMIP. PA significantly induced CMIP expression in the control siRNA-expressing podocytes, but not in the siCMIP-expressing cells (Fig. 2A). In non-PA-treated podocytes, CMIP knockdown exhibited no obvious effect on the p-cofilin-1 ${ }^{\text {Ser3 }}$ expression level. However, PA-induced upregulation of the $\mathrm{p}$-cofilin- ${ }^{\text {Ser3 }}$ expression level was significantly prevented in siCMIP-expressed podocytes (Fig. 2B). Therefore, the current in vivo and in vitro results demonstrate that stimulation of CMIP expression decreased the activity of cofilin-1. Cofilin is an actin-binding protein that is essential for actin de-polymerization (13). Subsequently, the effect of CMIP on actin remodeling in PA-treated podocytes was evaluated in the current study. CMIP knockdown alone did not alter the architecture of the actin cytoskeleton. Compared with non-PA-treated cells, the application of PA led to a marked re-organization of the actin cytoskeleton, which was displayed as nucleation and loss of actin filaments, which was prevented in the CMIP knockdown podocytes, but not in the control knockdown cells (Fig. 2C). The average length (mean \pm standard deviation) of actin filaments was further measured and compared, demonstrating that the length 
A

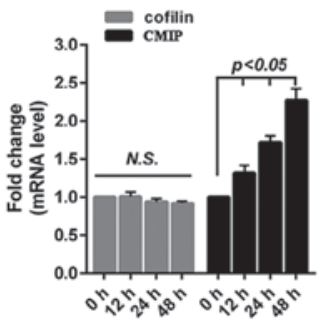

E

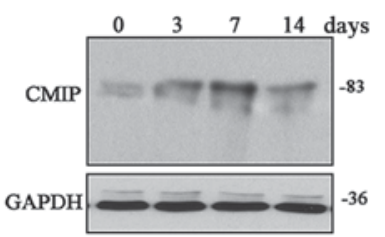

B

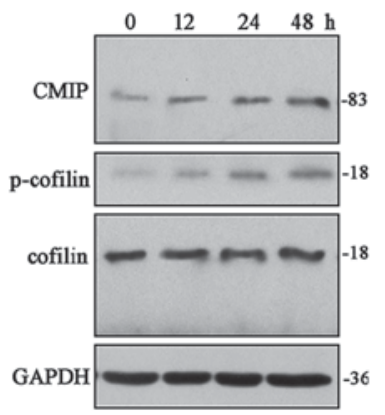

$\mathrm{C}$

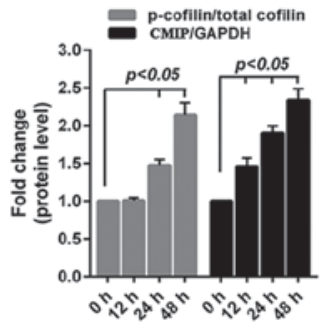

F

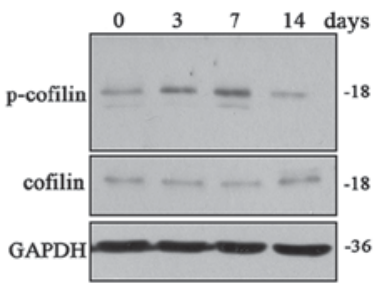

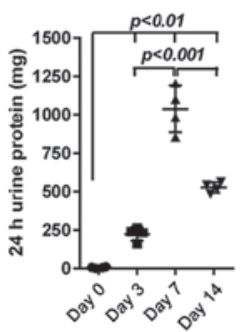

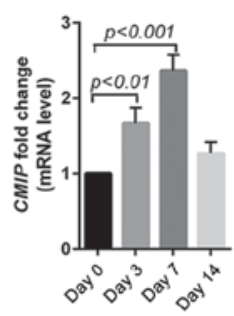

D
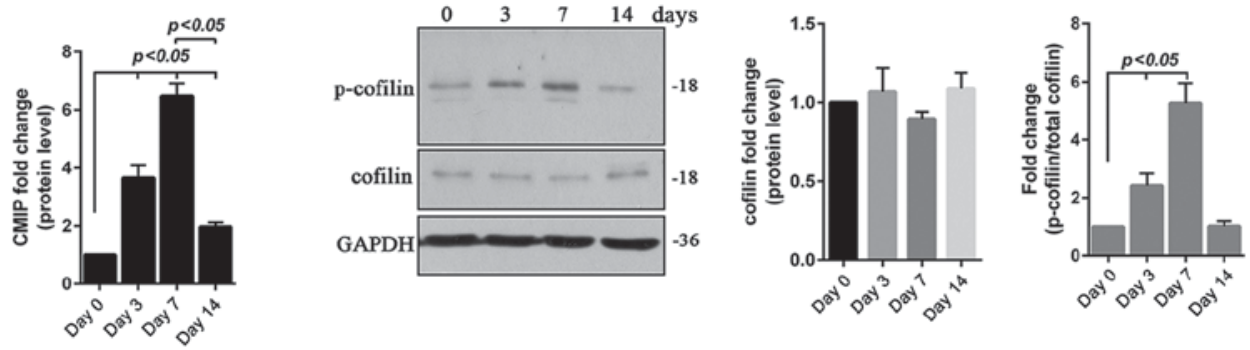

Figure 1. Expression levels of p-cofilin-1 and CMIP are increased following PA-induced podocyte damage. Mouse podocytes were treated with PA (75 $\mu \mathrm{g} / \mathrm{ml})$ for different time periods. (A) Quantitative polymerase chain reaction was used to assess the expression levels of CMIP and cofilin-1. (B) The abundance of CMIP and cofilin-1, as well as p-cofilin-1 was assessed using immunoblot assay ( $\mathrm{n}=3$ independent experiments and the representative blots are presented) (C) Nephropathy was established in rats by intraperitoneal injection with PA (10 mg/100 g body weight). The 24-h urine was collected at the indicated time points, and proteinuria was measured and compared. Isolated glomeruli were used for RNA and protein extraction. The abundance of CMIP (D) mRNA and (E) protein was analyzed. (F) The expression level of total cofilin-1 and p-cofilin-1 was examined using immunoblot assay ( $\mathrm{n}=4$ rats per time point and the representative blots are shown). P, phosphorylated; CMIP, c-Maf inducing protein; PA, puromycin aminonucleoside; n.s., not significant.

A

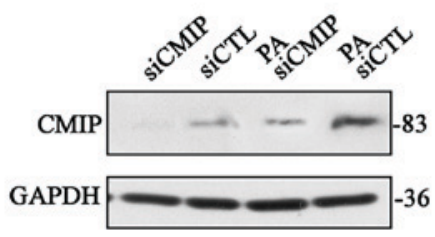

C

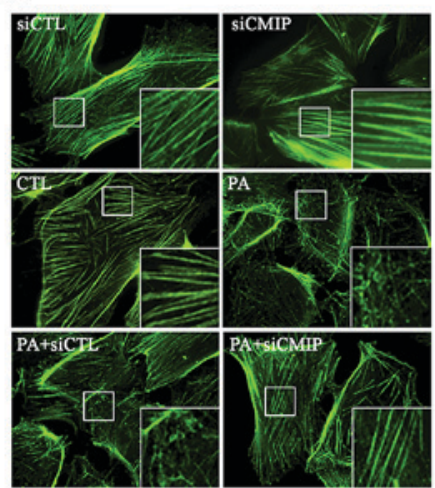

B
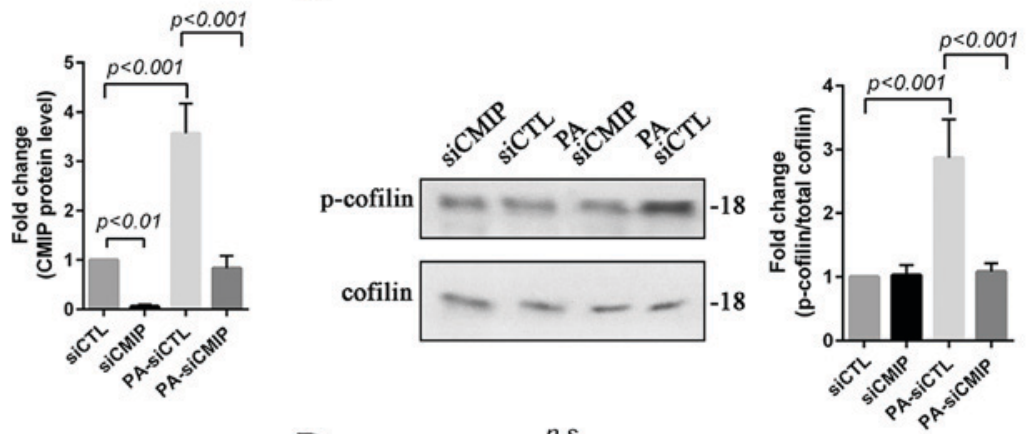

D

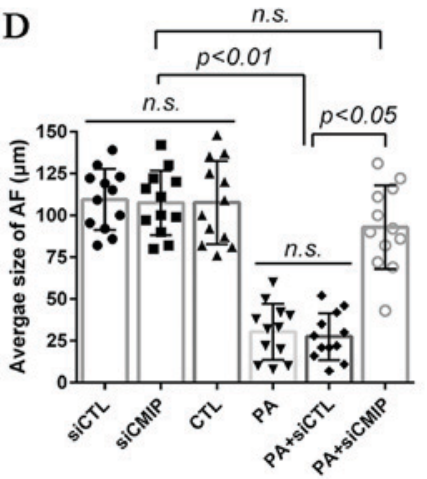

Figure 2. Stimulation of CMIP expression decreased cofilin-1 activity and induced actin reorganization in PA-treated podocytes. Mouse podocytes with stable knockdown of CMIP (siCMIP) were treated with PA $(75 \mu \mathrm{g} / \mathrm{ml})$ for $24 \mathrm{~h}$. Podocytes expressing control siRNA served as the knockdown control (siCTL). Total cellular protein was extracted using RIPA buffer. The abundance of (A) CMIP, and (B) cofilin-1 and p-cofilin-1 was assessed by immunoblot assay $(n=3$ independent experiments and the representative blots are presented). Cells were fixed and permeabilized, and the actin cytoskeleton was revealed using Alexa 488-conjugated phalloidin. (C) The representative images are presented (magnification, x20). (D) The average length of actin filaments was measured with ImageJ and compared. Ten cells were analyzed per group. CMIP, c-Maf inducing protein; PA, puromycin aminonucleoside; p, phosphorylated; n.s., not significant; si, small interferring; CTL, control. 
A

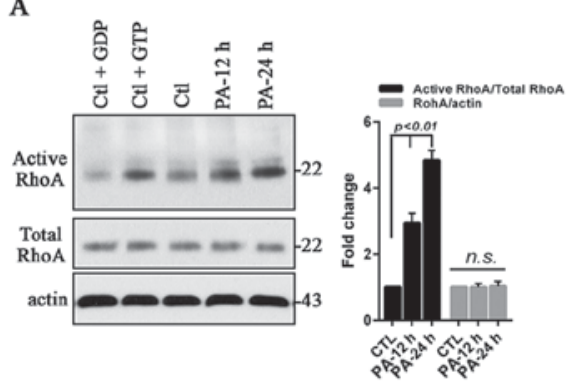

B

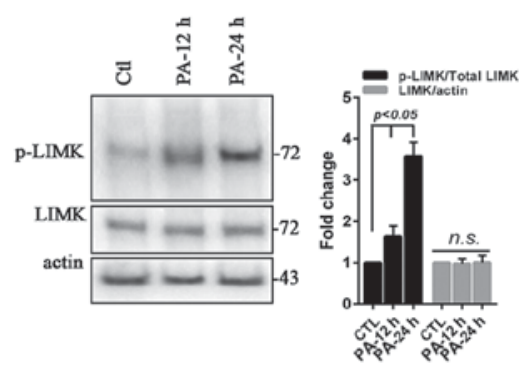

E

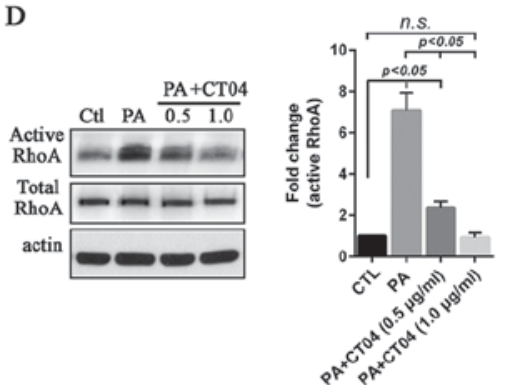

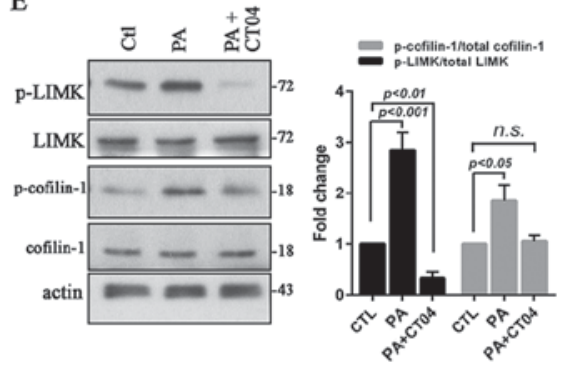

$\mathrm{C}$

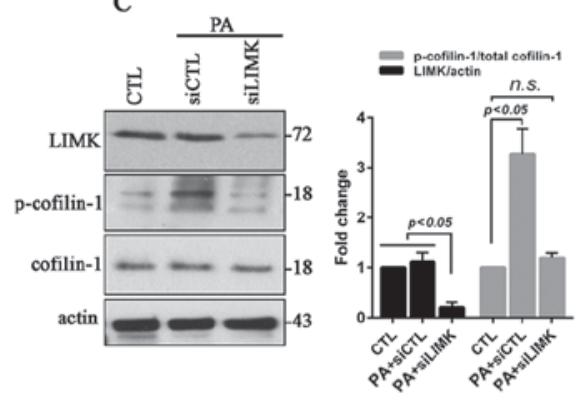

Figure 3. RhoA activation is responsible for reduction of cofilin-1 activity in PA-treated podocytes. Mouse podocytes were treated with PA (75 $\mu \mathrm{g} / \mathrm{ml})$ for 12 and $24 \mathrm{~h}$. Total cellular protein was extracted using RIPA lysis buffer. Pull down assay with agarose bead-conjugated Rhotekin-Raf-like Ras-binding domain protein was used to quantify RhoA activation. (A) GDP- and GTP $\gamma$ S-treated samples served as the negative and positive control, respectively. (B) The abundance of LIMK and p-LIMK was examined via immunoblot assay. (C) Mouse podocytes with stable knockdown of LIMK (siLIMK) were treated with PA $(75 \mu \mathrm{g} / \mathrm{ml})$ for $24 \mathrm{~h}$. Podocytes expressing control siRNA served as the knockdown control (siCTL). The abundance of LIMK, as well as cofilin-1 and p-cofilin-1 was assessed via immunoblotting. Mouse podocytes were pre-treated with Rho inhibitor, CT04 (final concentration, $0.5 \mathrm{and} 1.0 \mu \mathrm{g} / \mathrm{ml}$ ) for $1 \mathrm{~h}$. Cells were subsequently treated with PA $(75 \mu \mathrm{g} / \mathrm{ml})$ for $24 \mathrm{~h}$ in the presence of CT04. (D) The RhoA activation level was evaluated using pull down assay. The abundance of (E) p-LIMK and p-cofilin-1 was examined via immunoblotting ( $\mathrm{n}=3$ independent experiments and the representative blots are presented). RhoA, Ras homolog gene family, member A; PA, puromycin aminonucleoside; GDP, guanosine diphosphate; GTP, guanosine-5'-triphosphate; LIMK, LIM domain kinase; p, phosphorylated; n.s., not significant; si, small interferring; CTL, control.

of actin filaments was significantly decreased $(\mathrm{P}<0.01)$ in PA-treated podocytes, which was protected by siCMIP, but not by siCTL (Fig. 2D).

Signaling from RhoA activation is responsible for upregulation of p-cofilin-1 in PA-treated podocytes. Previous studies reported that the small GTPase, RhoA is crucial in regulating the activity of cofilin-1 (17). The level of activated RhoA was evaluated in PA-treated podocytes, and it was identified that, when compared with the control, the activated RhoA level was significantly increased at 12 and $24 \mathrm{~h}$ following PA administration (Fig. 3A; $\mathrm{P}<0.01)$. Consistently, the $\mathrm{p}-\mathrm{LIMK}^{\mathrm{Thr} 508}$, a downstream effector of RhoA, increased significantly in the PA-treated podocytes (Fig. 3B; $\mathrm{P}<0.01$ ). It was demonstrated that $\mathrm{RhoA}$ regulates cofilin-1 activity via LIMK (18). In addition, it was found that PA-induced upregulation of $\mathrm{p}$-cofilin-1 ${ }^{\text {Ser3 }}$ was reduced significantly by LIMK knockdown (Fig. 3C; $\mathrm{P}<0.01$ ). The role of RhoA signals was further investigated using RhoA inhibitor, CT04. Two different concentrations $(0.5$ or $1.0 \mathrm{mg} / \mathrm{ml})$ of CT04 were used in the current study. In cultured podocytes, the two significantly blocked PA-induced RhoA activation (Fig. 3D; $\mathrm{P}<0.01)$. The effects of CT04, at a concentration of $1.0 \mathrm{mg} / \mathrm{ml}$, on LIMK and cofilin-1 activities were investigated. The present data indicated that CT04 prevented the increase of $\mathrm{p}-\mathrm{LIMK}^{\mathrm{Th} 508}$ and p-cofilin-1 ${ }^{\text {Ser3 }}$ in PA-treated podocytes (Fig. 3E).

Stimulation of CMIP expression results in RhoA activation in PA-treated podocytes. In CMIP knockdown podocytes,
RhoA activity was investigated following PA treatment for 24 h. Firstly, knockdown of CMIP was confirmed in PA-treated podocytes. The immunoblotting results indicate that PA-induced CMIP expression was not detected in cultured podocytes with CMIP knockdown (Fig. 4A). PA-induced RhoA activation was prevented by CMIP knockdown in the cultured podocytes (Fig. 4B), indicating that overproduced CMIP may cause RhoA activation. Furthermore, CMIP knockdown significantly reduced the p-LIMK ${ }^{\text {Thr508 }}$ level in PA-treated podocytes (Fig. 4C; $\mathrm{P}<0.05$ ). It is well known that Rho GTPase activity is regulated by numerous guanine nucleotide exchange factors and GTPase-activating proteins (19). The current study identified that the phosphorylated level of p190RhoGAP at Tyr1105, also termed GRF1, was significantly decreased in the PA-treated podocytes compared with the untreated cells (Fig. 4D; P<0.05), while PA-induced reduction of $\mathrm{p}-\mathrm{GRF} 1^{\mathrm{Ty} 1105}$ was significantly prevented by CMIP knockdown (Fig. 4D; P<0.05). In untreated podocytes, CMIP was overexpressed by transfection of pcDNA-CMIP plasmids. In comparison with the control plasmid transfection, decreased p-GRF1 $1^{\text {Tyr1 } 105}$ and increased RhoA activation was detected in CMIP overexpressed podocytes (Fig. 4E). These results indicate that PA-induced overproduction of CMIP leads to RhoA activation potentially by inhibiting p190RhoGAP.

Downregulation of the activity of Src family kinase Fyn is mediated by overproduced CMIP in PA-treated podocytes. It has been reported that increased CMIP inhibits the activation 
A
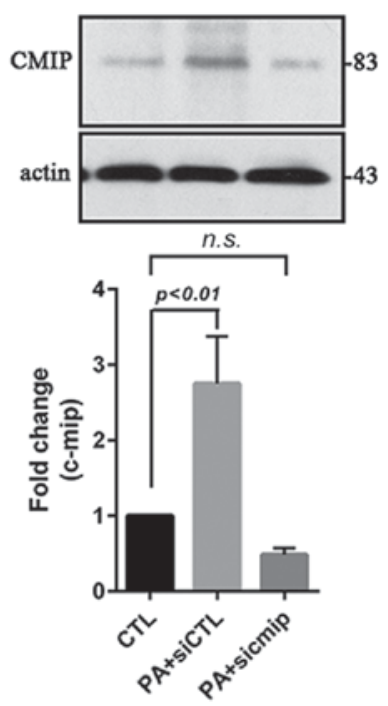

D

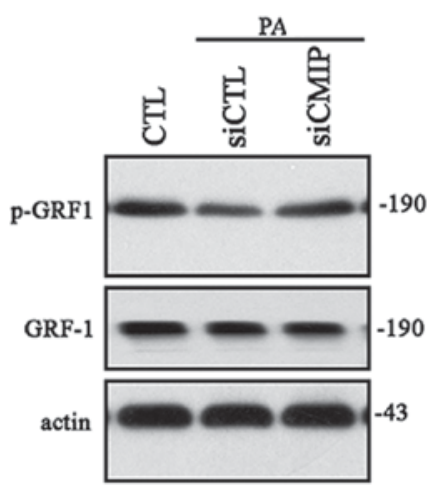

B
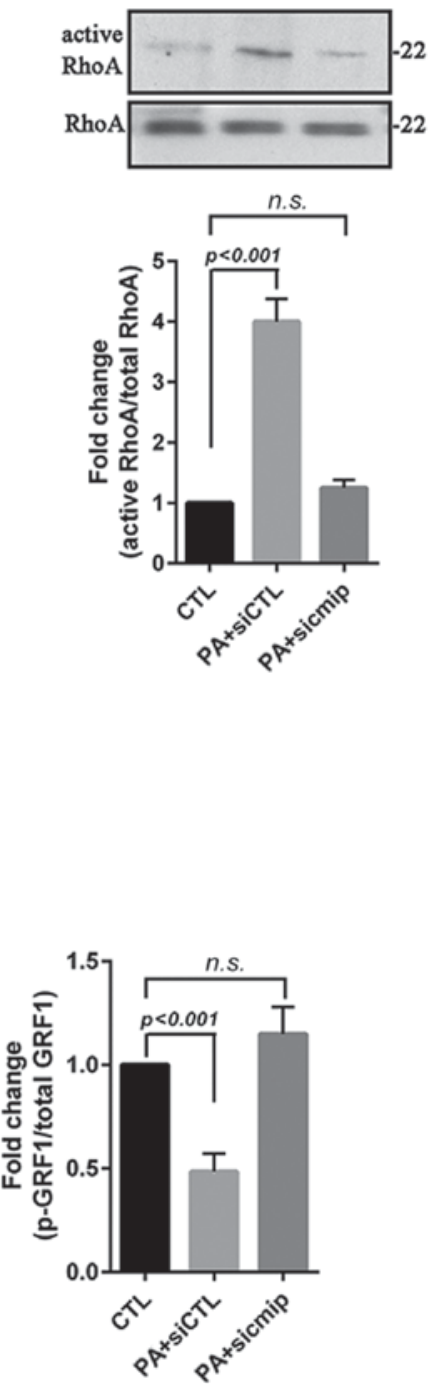

C
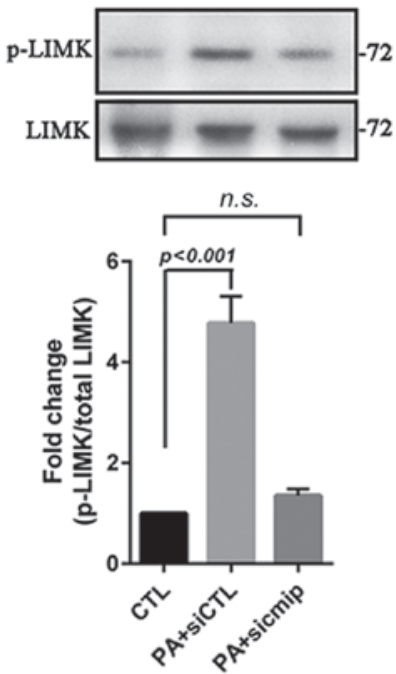

$\mathrm{E}$

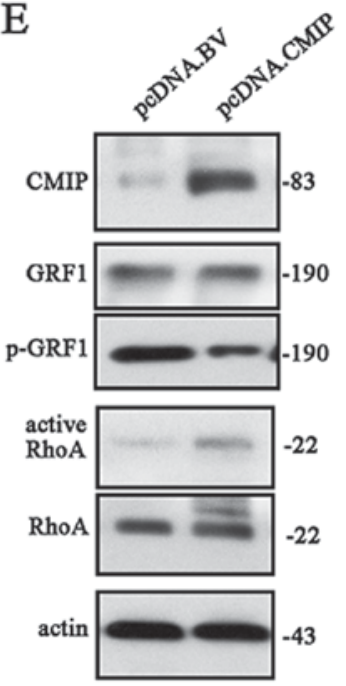

Figure 4. Overproduced CMIP leads to activation of RhoA signals in PA-treated podocytes. Mouse podocytes with stable knockdown of CMIP (siCMIP) were treated with PA $(75 \mu \mathrm{g} / \mathrm{ml})$ for $24 \mathrm{~h}$. Podocytes expressing control siRNA served as a knockdown control (siCTL). Total cellular protein was extracted using RIPA lysis buffer. (A) Knockdown of CMIP was confirmed via immunoblot assay. (B) Pull down assay with agarose bead-conjugated Rhotekin-Raf-like Ras-binding domain protein was performed to quantify RhoA activation. The expression levels of (C) p-LIMK and (D) p-GRF1 were examined. (E) Overexpression of CMIP in podocytes was obtained by transient transfection of pcDNA-CMIP. Equal quantities of BV transfection served as a control. The p-GRF1 and RhoA activation levels were assessed ( $\mathrm{n}=3$ independent experiments, and the representative blots are presented). CMIP, c-Maf inducing protein; RhoA, Ras homolog gene family, member A; PA, puromycin aminonucleoside; LIMK, LIM domain kinase; GRF1, growth regulating factor 1; BV, blank vector; n.s., not significant; si, small interferring; CTL, control.

of the Src kinase, Fyn in CMIP-overexpressed podocytes and in angiotensin II-induced podocyte damage $(5,20)$. The activation status of the Src kinase Fyn is associated with RhoA activity. Src activation leads to the inactivation of RhoA through formation of a complex with p190RhoGAP and mDia interacting protein (21). In PA-treated podocytes, the activation level of Fyn, a predominant Src kinase in podocytes, was analyzed. Overproduction of CMIP was observed in PA-treated podocytes. Therefore, the activity of the Src kinase was investigated in a PA-induced podocyte damage model. The application of PA significantly decreased $(\mathrm{P}<0.05)$ the activated level of Src kinase $\left(\mathrm{p}-\mathrm{Src}^{\mathrm{Tyr} 418}\right)$, while the inactivated level $\left(\mathrm{p}-\mathrm{Src}^{\mathrm{Tyr} 527}\right)$ was increased in the cultured podocytes (Fig. 5A). The specific activity of Fyn was evaluated using an immunoprecipation assay with an anti-Fyn antibody and blotting with anti-pSrc418 antibody. The PA-induced reduction of activated Src kinase $\left(\mathrm{p}-\mathrm{Src}^{\mathrm{Tyr} 418}\right.$ ) was prevented in CMIP knockdown podocytes as compared with the control knockdown cells (Fig. 5B).

\section{Discussion}

Expression of CMIP, a novel podocyte protein, is induced in human and murine minimal change disease and membranous nephropathy, and in angiotensin II, lipopolysaccharide and ADR-induced podocyte damage $(5,8,9,20)$. In the present study, CMIP expression was examined in a common podocyte damage model induced by PA. The results indicated that CMIP expression was induced in a time-dependent manner 
A

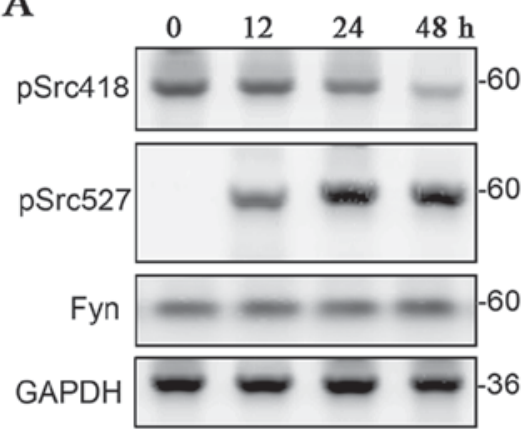

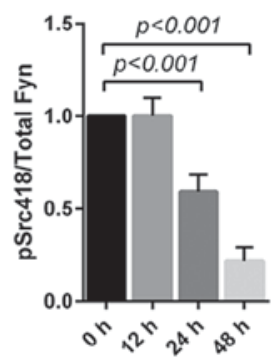

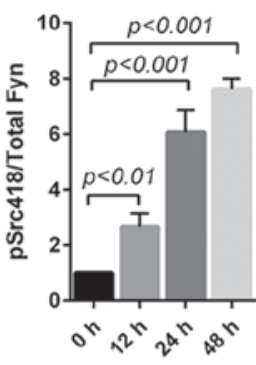

B

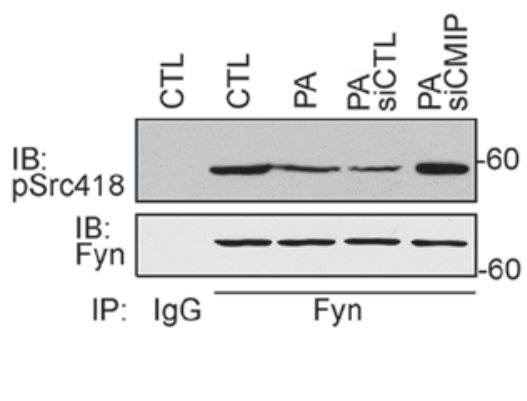

Figure 5. Stimulation of CMIP expression prevents the activation of Src kinase, Fyn in PA-treated podocytes. (A) Mouse podocytes were treated with PA $(75 \mu \mathrm{g} / \mathrm{ml})$ for the indicated time points. Total cellular protein was extracted using RIPA lysis buffer. The abundance of p-Src at Tyr418 and at Tyr527, as well as total Src kinase, Fyn was examined via immunoblot assay ( $\mathrm{n}=3$ independent experiments, and the representative blots are presented). (B) Mouse podocytes with stable knockdown of CMIP (siCMIP) were treated with PA $(75 \mu \mathrm{g} / \mathrm{ml})$ for $24 \mathrm{~h}$. Podocytes expressing control siRNA served as knockdown controls (siCTL). The specific activation level of Fyn was evaluated via immunoprecipitation assay with anti-Fyn antibody followed by blotting with anti-pSrc418 antibody. Normal IgG served as a control. CMIP, c-Maf inducing protein; PA, puromycin aminonucleoside; IgG, immunoglobulin G; IB, immunoblotting; IP, immunoprecipitation.

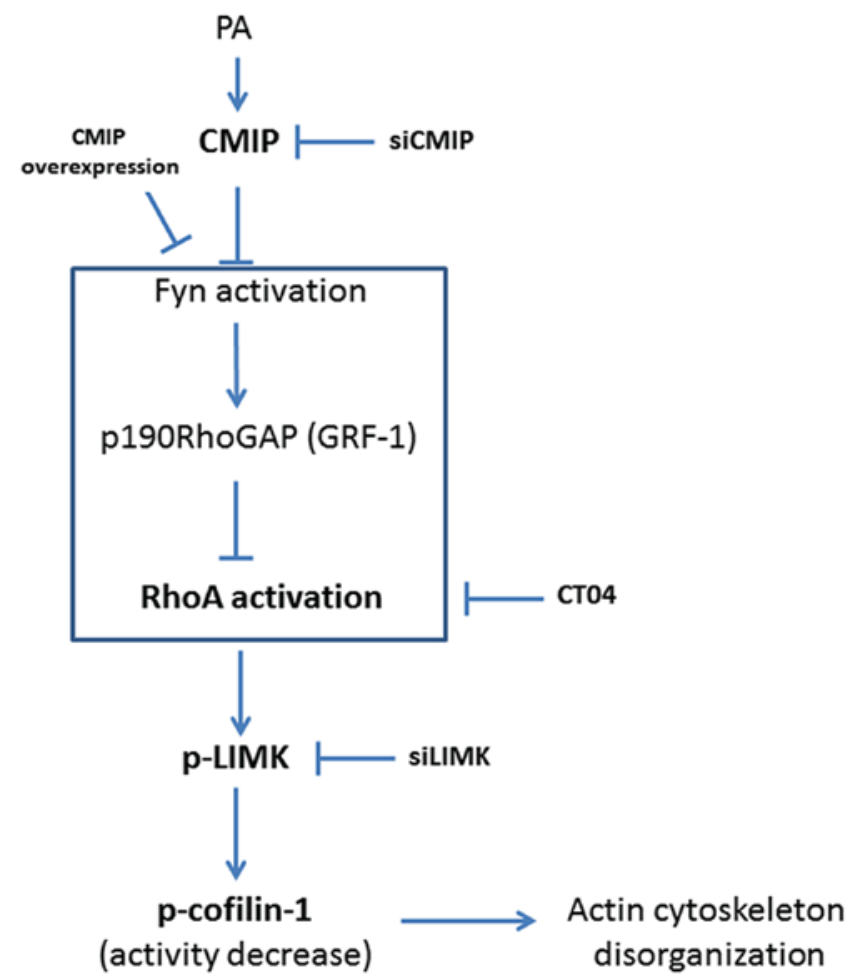

Figure 6. Schematic of the CMIP and cofilin signaling pathway associated with PA-induced podocyte damage. As indicated in the boxed region, the Src kinase Fyn inhibits RhoA activation by enhancing p190RhoGAP (GRF-1). In podocytes, stimulation of CMIP expression by PA, or overexpression of CMIP, decreases the Fyn activity, which leads to activation of RhoA and increases phosphorylation of downstream effector, LIMK, resulting in an increase of p-cofilin while reducing cofilin activity. The decreased cofilin activity induces actin cytoskeleton disorganization. Inhibition of RhoA activation by knockdown of CMIP (siCMIP) or its inhibitor, CT04 prevents reduction of cofilin activity and thus preserves the actin architecture. In addition, direct knockdown of RhoA downstream effector, LIMK (siLIMK) increases cofilin activity. CMIP, c-Maf inducing protein; PA, puromycin aminonucleoside; GRF-1, growth regulating factor 1; RhoA, Ras homolog gene family, member A; LIMK, LIM domain kinase; P, phosphorylated.

following PA applications in vitro and in vivo. Notably, in a PA-induced nephropathy rat, an increased CMIP expression level preceded peak proteinuria, indicating that induction of
CMIP expression may be significant in initiating podocyte damage and proteinuria occurrence.

Cofilin-1 is a cytoskeletal protein, which controls actin de-polymerization $(10,11)$. Obvious changes in total cofilin-1 expression levels were not observed, whereas time-dependent increases of p-cofilin-1 on serine 3 were detected. Phosphorylation of cofilin-1 on serine 3 leads to reduction of actin binding and depolymerizing activity $(11,12)$. Notably, a PA-induced increase of p-cofilin-1 was prevented by CMIP knockdown in cultured podocytes. To further identify the role of CMIP in PA damaged podocytes, alterations of the actin cytoskeleton were further assessed with phalloidin staining, as it has been shown that CMIP and cofilin are critical in regulating the remodeling of the actin cytoskeleton in podocytes. In PA-treated podocytes, it was reported that cyclosporine A protects podocytes via stabilization of cofilin-1 expression in the unphosphorylated state (22). The application of PA resulted in disorganization of actin and decreases in actin filament length, which was markedly prevented by CMIP knockdown. Therefore, the current data indicate that overproduced CMIP may be responsible for actin disorganization at least via downregulation of cofilin-1 activity in PA-induced podocyte injury. Notably, cofilin as an essential, conserved, small actin-binding protein has various roles in cytokinesis, endocytosis, embryonic development, stress responses and tissue regeneration (23). Therefore, evaluation of more podocyte damage markers, such as apoptosis, adhesion, and motility may be required to broaden understanding of the role of CMIP and cofilin in the induction of podocyte injury. However, p-cofilin at Ser3 also regulates cofilin translocation from the nucleus to the cytoplasm (24). In the current PA-mediated podocyte injury model, the localization or distribution changes of $\mathrm{p}$-cofilin require further investigation.

The small GTPase, RhoA increases the phosphorylation level of cofilin-1 through activation of LIMK (18). The current study found that RhoA activity and the level of p-LIMK were increased significantly in cultured podocytes following PA treatment. LIMK belongs to the actin-binding kinases, which phosphorylate members of the cofilin family of actin binding and filament severing proteins (18). In the present study, 
LIMK knockdown inhibited the increase of p-cofilin-1 level in PA-treated podocytes, indicating that RhoA-mediated LIMK activation is involved in the reduction of cofilin-1 activity in PA-damaged podocytes. The role of RhoA signaling cascades was further investigated using the cell permeable Rho inhibitor, CT04. CT04 is powerful for efficient inactivation of Rho GTPase in a variety of cultured cells (25). In the present study, PA-induced activation of RhoA was prevented by CT04. In PA-treated podocytes, inhibition of RhoA by CT04 decreased the phosphorylation level of LIMK and cofilin-1. Thus, these data indicate that the RhoA-mediated LIMK and cofilin-1 signaling was altered in PA-injured podocytes. The three small RhoGTPases, RhoA, cell division control protein 42 homolog (Cdc42), and Ras-related C3 botulinum toxin substrate 1 (Rac1), induce actin remodeling that is necessary for polymerization and de-polymerization via various major downstream effectors, including Rho kinase (ROCK), p21 activated kinase (Pak), LIMK and myosin light chain (MLC) (26). Although RhoA-ROCK and the Rac/Cdc42-Pak signaling pathway each has a unique substrate, evidence indicates that they modulate LIMK-cofilin activity, serving as a common downstream signal to regulate actin turnover (26). Therefore, further investigation is required if RhoA signaling directly affects cofilin activity following podocyte injury or if other small GTPases, such as Cdc42 and Rac1 are involved in this process.

It has been described that CMIP inhibits Src kinase activity in podocytes $(5,20)$. Src kinase family is a family of non-receptor tyrosine kinases, including Src, Yes, Fyn, Lyn and Lck $(27,28)$. In podocytes, Fyn is critical in regulating nephrin phosphorylation, and stimulation of CMIP expression downregulates nephrin phosphorylation by reducing Fyn activity $(5,20)$. Src kinase inhibits RhoA activation by enhancing the activity of p190RhoAGAP (GRF1) (19). In PA-treated podocytes, the present results indicate that overproduced CMIP is responsible for reduction of Fyn activity, as the reduced active form (Src418) and increased inactive form (Src527) were detected. The immunoprecipitation assay demonstrated that knockdown of CMIP specifically increased the level of activated Fyn. Consistently, a reduction of p-GRF1 was detected in PA-treated podocytes, which was prevented by CMIP knockdown. Furthermore, increased RhoA activity and its downstream effector, p-LIMK were prohibited by CMIP knockdown in podocytes upon treatment with PA. Additionally, the level of p-GRF1 was reduced, while RhoA activity was increased in podocytes overexpressing CMIP. Therefore, stimulation of CMIP expression by PA appeared to activate RhoA signals by inhibiting Fyn activity, leading to decreased p190RhoAGAP. Although overexpressed CMIP increased RhoA activity in podocytes, it requires further investigation to identify whether CMIP directly regulates RhoA activity in PA-induced podocyte damage.

In conclusion, the present findings indicate that CMIP inhibits cofilin-1 activity and alters podocyte cytoskeleton organization at least through RhoA signaling in PA-induced podocyte injury (Fig. 6). Induction of CMIP expression has also been detected in ADR nephropathy mice (9). It may be that this signaling pathway also occurs in ADR-induced podocyte damage. However, whether this is a common phenomenon or is applicable to other podocyte injury models requires investigation to elucidate further the function of the CMIP and cofilin signaling pathway in the development of podocyte damage.

\section{Acknowledgements}

The present study was supported by the National Natural Science Foundation of China (grant no. 81300577).

\section{References}

1. Scott RP and Quaggin SE: Review series: The cell biology of renal filtration. J Cell Biol 209: 199-210, 2015.

2. Cellesi F, Li M and Rastaldi MP: Podocyte injury and repair mechanisms. Curr Opin Nephrol Hypertens 24: 239-244, 2015.

3. Saleem MA: One hundred ways to kill a podocyte: Nephrol Dial Transplant 30: 1266-1271, 2015.

4. New LA, Martin CE and Jones N: Advances in slit diaphragm signaling. Curr Opin Nephrol Hypertens 23: 420-430, 2014.

5. Zhang SY, Kamal M, Dahan K, Pawlak A, Ory V, Desvaux D, Audard V, Candelier M, BenMohamed F, Matignon M, et al: C-mip impairs podocyte proximal signaling and induces heavy proteinuria. Sci Signal 3: ra39, 2010.

6. Reiser J and Mundel P: Danger signaling by glomerular podocytes defines a novel function of inducible B7-1 in the pathogenesis of nephrotic syndrome. J Am Soc Nephrol 15: 2246-2248, 2004.

7. Trimarchi H: Abatacept and glomerular diseases: The open road for the second signal as a new target is settled down. Recent Pat Endocr Metab Immune Drug Discov 9: 2-14, 2015.

8. Sendeyo K, Audard V, Zhang SY, Fan Q, Bouachi K, Ollero M, Rucker-Martin C, Gouadon E, Desvaux D, Bridoux F, et al: Upregulation of C-mip is closely related to podocyte dysfunction in membranous nephropathy. Kidney Int 83: 414-425, 2013.

9. Liu Y, Su L, Lin Q, Han Y, You P and Fan Q: Induction of C-mip by IL-17 Plays an important role in adriamycin-induced podocyte damage. Cell Physiol Biochem 36: 1274-1290, 2015.

10. Spence EF and Soderling SH: Actin out: Regulation of the synaptic cytoskeleton. J Biol Chem 290: 28613-28622, 2015.

11. Kanellos G and Frame MC: Cellular functions of the ADF/cofilin family at a glance. J Cell Sci 129: 3211-3218, 2016.

12. Garg P, Verma R, Cook L, Soofi A, Venkatareddy M, George B, Mizuno K, Gurniak C, Witke W and Holzman LB: Actin-depolymerizing factor cofilin-1 is necessary in maintaining mature podocyte architecture. J Biol Chem 285: 22676-22688, 2010.

13. Berger K and Moeller MJ: Cofilin-1 in the podocyte: A molecular switch for actin dynamics. Int Urol Nephrol 43: 273-275, 2011.

14. Teng B, Lukasz A and Schiffer M: The ADF/cofilin-pathway and actin dynamics in podocyte injury. Int J Cell Biol 2012: 320531, 2012.

15. Guan N, Ding J, Deng J, Zhang J and Yang J: Key molecular events in puromycin aminonucleoside nephrosis rats. Pathol Int 54: 703-711, 2004.

16. Livak KJ and Schmittgen TD: Analysis of relative gene expression data using real-time quantitative PCR and the 2(-Delta Delta C(T)) method. Methods 25: 402-408, 2001.

17. Lin T, Zeng L, Liu Y, DeFea K, Schwartz MA, Chien S and Shyy JY: Rho-ROCK-LIMK-cofilin pathway regulates shear stress activation of sterol regulatory element binding proteins. Circ Res 92: 1296-1304, 2003.

18. Qiu Y, Chen WY, Wang ZY, Liu F, Wei M, Ma C and Huang YG: Simvastatin attenuates neuropathic pain by inhibiting the RhoA/LIMK/cofilin pathway. Neurochem Res 41: 2457-2469, 2016.

19. Duman JG, Mulherkar S, Tu YK, X Cheng J and Tolias KF: Mechanisms for spatiotemporal regulation of Rho-GTPase signaling at synapses. Neurosci Lett 601: 4-10, 2015.

20. Yu L, Lin Q, Feng J, Dong X, Chen W, Liu Q and Ye J: Inhibition of nephrin activation by C-mip through Csk-Cbp-Fyn axis plays a critical role in Angiotensin II-induced podocyte damage. Cell Signal 25: 581-588, 2013.

21. Meng W, Numazaki M, Takeuchi K, Uchibori Y, Ando-Akatsuka Y, Tominaga M and Tominaga T: DIP (mDia interacting protein) is a key molecule regulating Rho and Rac in a Src-dependent manner. EMBO J 23: 760-771, 2004. 
22. Li X, Zhang X, Li X, Wang X, Wang S and Ding J: Cyclosporine A protects podocytes via stabilization of cofilin-1 expression in the unphosphorylated state. Exp Biol Med (Maywood) 239: 922-936, 2014.

23. Condeelis $\mathrm{J}$ : How is actin polymerization nucleated in vivo? Trends Cell Biol 11: 288-293, 2001.

24. Nebl G, Meuer SC and Samstag Y: Dephosphorylation of serine 3 regulates nuclear translocation of cofilin. J Biol Chem 271: 26276-26280, 1996

25. Filina JV, Gabdoulkhakova AG and Safronova VG: RhoA/ROCK downregulates FPR2-mediated NADPH oxidase activation in mouse bone marrow granulocytes. Cell Signal 26: 2138-2146, 2014.
26. Wang $\mathrm{W}$ and Townes-Anderson E: Lim kinase, a bi-functional effector in injury-induced structural plasticity of synapses. Neural Regen Res 11: 1029-1032, 2016.

27. Chuang PY and He JC: Signaling in regulation of podocyte phenotypes. Nephron Physiol 111: p9-p15, 2009.

28. Roskoski R Jr: Src protein-tyrosine kinase structure, mechanism, and small molecule inhibitors. Pharmacol Res 94: 9-25, 2015. 